$R M x A C, \mathbf{5 3}, 14-17(2021)$

(C) 2021: Instituto de Astronomía, Universidad Nacional Autónoma de México

https://doi.org/10.22201/ia.14052059p.2021.53.04

\title{
TECHNICAL AND SOFTWARE UPGRADES COMPLETED AND PLANNED AT OARPAF
}

\author{
D. Ricci ${ }^{1}$, L. Cabona ${ }^{2,3}$, A. La Camera ${ }^{4}$, C. Righi ${ }^{2,5}$, F. Nicolosi ${ }^{6}$, and S. Tosi ${ }^{5,6}$
}

\section{RESUMEN}

Presentamos actualizaciones técnicas, instrumentales y de software, desarrolladas y planeadas en el observatorio astronómico denominado Osservatorio Astronomico Regionale Parco Antola, Fascia (OARPAF), que alberga un telescopio alt-azimutal Cassegrain-Nasmyth de $80 \mathrm{~cm}$. El observatorio, ubicado en los Apeninos de Liguria, actualmente puede ser operado ya sea para uso científico por profesionales (con cámara fotométrica) o para observaciones por aficionados (usando oculares), simplemente cambiando el espejo terciario entre los dos focos Nasmyth de manera manual. Los principales campos de observación científica están relacionados con tránsitos de exoplanetas, cuásares y lentes gravitacionales relacionadas con cuásares, habiéndose publicado resultados recientemente. Una estrategia de remotización y robotización del observatorio ha permitido configurar toda la estructura (telescopio, cúpula, instrumentos, sensores y aparatos de monitorización) implementándose constantes mejoras. Presentamos en este trabajo las actualizaciones vigentes, principalmente relacionadas con lo que concierne al aspecto hardware de la robotización de la cúpula; en cuanto al aspecto de instrumentación, se describe así como un nuevo soporte modular para instrumentción con capacidades espectrofotométricas, que se encuentra en una fase de diseño preliminar, pudiendo mejorar el rendimiento del telescopio y ampliando los potenciales campos científicos de investigación. En este marco, ha comenzado la adquisición de material espectrofotométrico. Por otro lado, en cuanto al software, se ha desarrollado un innovador software basado en web que, usando websockets y node.js ya se puede utilizar para controlar la cámara, y que se ampliará para poder administrar los otros instrumentos del observatorio y para el almacenamiento de la base de datos de imágenes.

\section{ABSTRACT}

We present technical, instrumental, and software upgrades completed and planned at astronomical observatory called "Osservatorio Astronomico Regionale Parco Antola, Fascia" (OARPAF), hosting an 80cm, alt-az Cassegrain-Nasmyth telescope. The observatory, located in the Ligurian Apennines, can currently be operated either for scientific (photometry camera) or amateur (ocular) observations, by switching the tertiary mirror between the two Nasmyth foci using a manual handle. The main scientific observational topics are related up to now to exoplanetary transits, QSOs, and gravitationally lensed quasars, and results are being recently published. A remotization and robotization strategy of the entire structure (telescope, dome, instruments, sensors and monitoring) have been set up and it is in progress. We report the current upgrades, mainly related for what concerns the "hardware" side to the robotization of the dome. On the instrumentation side, a new modular support for instruments with spectrophotometric capabilities is on a preliminary design phase, improving the telescope performances and broadening the potential science fields. In this framework, the procurement of spectrophotometric material has started. On the software side, an innovative web-based software relying on websockets and node.js can already be used to control the camera, and it will be extended to manage the other components of the instrument, of the observatory, and of the image database storage.

Key Words: instrumentation: detectors - telescopes

\footnotetext{
${ }^{1}$ INAF - Osservatorio Astronomico di Padova, Vicolo dell'Osservatorio 5, 35122 Padua, Italy.

${ }^{2}$ INAF - Osservatorio Astronomico di Brera, via Bianchi 46, 23807 Merate, Italy.

${ }^{3}$ Università degli Studi dell'Insubria, via Valleggio 11, 22100 Como, Italy.

${ }^{4}$ Teiga srls, Viale Brigate Partigiane 16, 16129 Genoa, Italy.

${ }^{5}$ Istituto Nazionale di Fisica Nucleare - Sezione di Genova, Via Dodecaneso 33, 16146 Genoa, Italy.
}

\section{OVERVIEW}

The astronomical observatory called "Osservatorio Astronomico Regionale Parco Antola, Fascia" (OARPAF), is realized in the Northern Italy, near Mt. Antola (Fig. 1), at $1450 \mathrm{~m}$ a.s.l. within the Antola Regional Reserve. The telescope is a Cassegrain-

\footnotetext{
${ }^{6}$ Dipartimento di Fisica - Università degli studi di Genova, Via Dodecaneso 33, 16146 Genoa, Italy.
} 


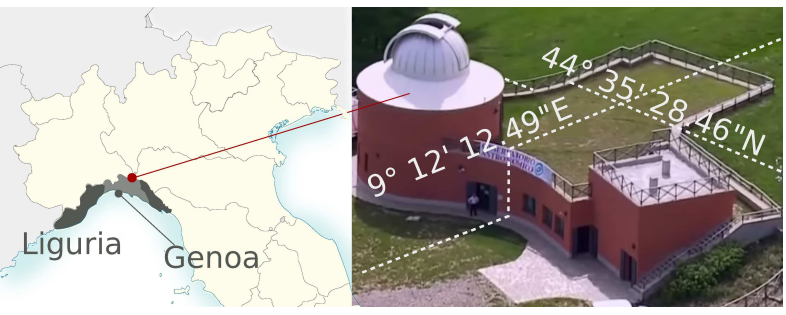

Fig. 1. Location of the $80 \mathrm{~cm}$ OARPAF telescope, at $1450 \mathrm{~m}$ a.s.l. within the Antola Regional Reserve. Coordinates are: $44^{\circ} 35,28.46 " \mathrm{~N}, 9^{\circ} 12$ ' $12.49 " \mathrm{E}$.

Nasmyth, with $80 \mathrm{~cm}$ of primary diameter, alt-az mounted, and designed by the Astelco company to foresee a double Nasmyth focal stations. The first one, provided with a field derotator, is dedicated to scientific observations; the second one is dedicated to ocular observations by amateurs (Federici et al. 2012). The scientific Nasmyth focus is currently equipped with an air-cooled SBIG STL 11000M camera, provided with an internal filter wheel with standard Johnson-Cousins UBVRI filters. Currently, the telescope is used for imaging/photometry by using this camera for several follow-up projects (Righi 2016; Ricci et al. 2017).

The observatory will be remotized in the next future. To reach this goal, a planned stop of the scientific activities is allowing to proceed with dome upgrades, telescope maintenance, and improvements of Local Area Network (LAN) and internet connection.

We also decided to substitute the current CCD device with a multi-functional instrument with superior capabilities, providing imaging/photometry, long slit spectroscopy, échelle spectroscopy and a "service" focal station for further implementation. Material to reach this goal is being procured, and the SBIG STL 11000M camera will be reused for the project.

The instrument control software based on modern web technologies in order to ease remote control, scalability, and maintenance.

In this proceedings we discuss the upgrades of the observatory $(\S 2)$, we describe the preliminary design of the new instrument ( $(3)$, and finally we give some detail of the instrument control software $(\S 4)$. Conclusions are given in $(\S 5)$.

\section{OBSERVATORY STATUS AND UPGRADES}

In recent years, the observatory suffered from several issues that limited its functionalities.

The dome was initially not foreseen for remotization. A custom solution based on Raspberry $\mathrm{Pi}+$ Arduino, with a command line Python script, is used as interface between the Telescope Control Software (TCS) and the dome to lock the rotation. However, this system do not control the eyelid, and recent bad weather conditions stressed the structure so that it also accounts with water infiltration. For all these reasons, a new waterproof dome linked to the TCS software, and with a doublymotorized eyelid security system is being was installed by the Gambato company and financed by the University of Genoa.

The Astelco company was also contacted in order to proceed with major maintenance activities: cleaning of the primary mirror, optical alignment, tests of the hydraulic pistons of the primary telescope petals. The proprietary TCS, Aste10S, will be also updated to the latest version. This update will be financed by the Antola Regional Reserve.

Malfunctions due to a thunderbolt affected the LAN switches, as well as unstable internet connection, and slowed down the remotization efforts. Moreover, the SBIG STL $11000 \mathrm{M}$ camera is powerconnected to an electric socket placed in the fork of the telescope, and USB-connected to a commercial pc inside the fork. This pc is accessed via Ethernet directly from a commercial router through a home network. In order to upgrade the network connections, the local administration allocated European and Regional funds to provide optical fiber and highspeed internet access to the municipalities and the public structures of the area surrounding the observatory. Moreover, the Antola Regional Reserve already procured the material for full implementation of a Gigabit LAN in the observatory. This update will be realized by the Liguria Digitale company. Unfortunately the current emergency situation delayed many of the foreseen interventions on the site of the Observatory and a full remotization could not be completed yet; it can be foreseen by the end of this year if no further delays intervene.

\section{INSTRUMENTATION STATUS AND UPGRADES}

Currently, scientific activities at OARPAF are carried out using the SBIG STL $11000 \mathrm{M}$ camera operating at the derotated Nasmyth focus. The decision on the opening / closing of the dome is based upon measurements provided by a DavisPro weather station equipped with pluviometer, anemometer, thermometer, barometer and humidity sensor. The observatory upgrades are the opportunity to improve scientific activities at OARPAF. To reach this goal, we plan to realize a new spectrophotometric instrument at the derotated Nasmyth focus. The pre- 


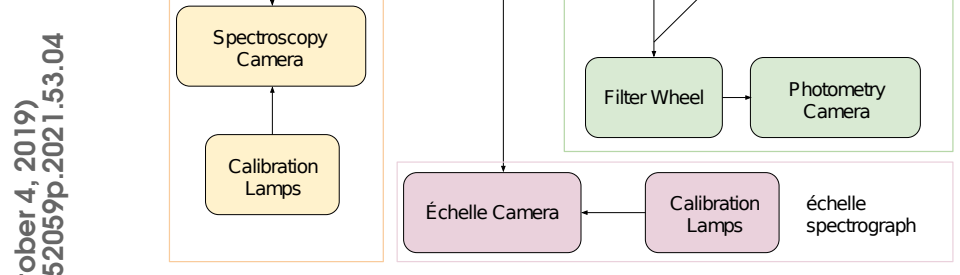

Fig. 2. Preliminary design of the new OARPAF instrument. The mode selector reflects the incoming light to one of the $3+1$ instruments: the imaging/photometry devices (in green, at the right), the échelle spectrograph (in pink, at the bottom), the long slit spectrograph (in yellow, at the left), and the "service" future instrument (in blue, in the top-left). A further implementation foresees a dome lamp for calibration.

liminary design foresees a flange interface, in order to provide $3+1$ focal stations via a linear motor mode selector provided with flat, $45^{\circ}$ mirrors: 1. imaging/photometry mode; 2. long slit spectroscopy mode; 3 . échelle spectroscopy mode; 4 . "service" mode. All configurations use the derotated, on axis, embedded guiding camera in the imaging/photometry path.

\subsection{Imaging/photometry mode}

This is the main use mode of the new instrument. The light proceeds on a straight path from the telescope until a SBIG AO-X Tip/Tilt lens able to act up to a $10 \mathrm{~Hz}$ rate. After this lens, the embedded, on axis SBIG STX-guider camera is placed just before the $50 \mathrm{~mm}$ SBIG FW-7 filter wheel provided with $U B V R I+H \alpha$ filters and a free position. This $648 \times 486,7.4 \mu \mathrm{m}$ pixel guiding camera, placed in a way that avoids vignetting, is provided with a $0.7 \times$ focal reducer, and is used to send offset information both to the telescope and to the Tip/Tilt lens, if on. Finally, the light focuses on the $4096 \times 4096,9 \mu \mathrm{m}$ pixels array SBIG STX 16801 camera with a Class 1 CCD for imaging and photometry on a $20^{\prime}$ Field of View.

\subsection{Long slit spectroscopy mode}

This mode requires a flat mirror of the mode selector to address the light on the slit of the LHIRES III spectrograph. A part of the light proceeds straight to the guiding camera on the imager and

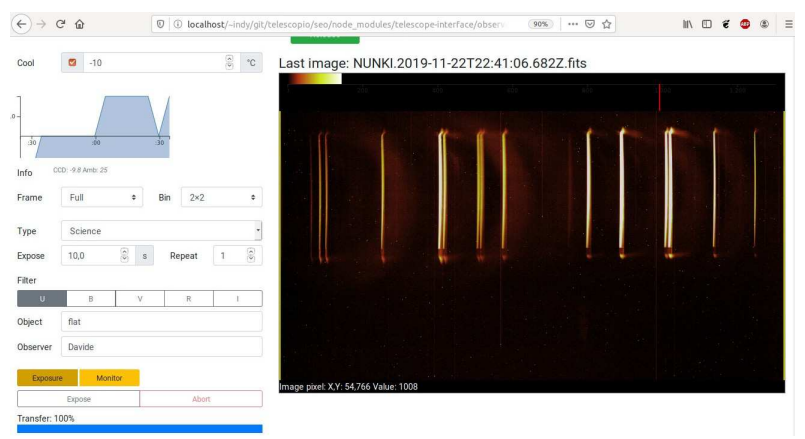

Fig. 3. Preliminary design of the web interface for the control of SBIG cameras. In this image, the SBIG STL $11000 \mathrm{M}$ camera is used to take a Neon-Argon lamp calibration frame for the LHIRES III.

it is not deviated by the flat mirror. The remaining light passes through the LHIRES III slit and the 1200 lines/mm diffraction grating, focusing a spectral range of $\approx 140 \mathrm{~nm}$ on the 4002 pixels of the SBIG STL $11000 \mathrm{M}$ camera, that will be reused. The central wavelength can be selected by using a micrometer screw that tilts the grating. This screw will be motorized in order to provide remote control. We foresee a resolution of $R \sim 5800$ in the visible band $(450-750 \mathrm{~nm})$. A standard spox module allows remote control of the embedded Neon-Argon calibration lamps.

\section{3. Échelle spectroscopy mode}

This mode requires a flat mirror to address the light on the head of a FLECHAS échelle spectrograph, containing a Th-Ar calibration lamp and supporting a $15 \mathrm{~m}$ long optical fiber. As in the case of the long slit spectroscopy mode, part of the light is foreseen to proceed straight to the guiding camera of the imager, while the light deviated by the flat mirror is focused on a ATIK 11000 camera on the FLECHAS at a $R \sim$ 9300 resolution.

\section{4. "Service" Mode}

This mode requires a flat mirror to address the light on focal station for generic purposes. Also in this case, part of the light is required to proceed straight to the guiding camera on the imager. The part of the light deviated by the flat mirror is focused on the service focal station, for further instrument implementations, maintenance operations, or, for example, astrophotography with a digital reflex camera.

\section{SOFTWARE STATUS AND UPGRADES}

OARPAF telescope is currently operated via ssh connection and web interface (see Fig. 3), 


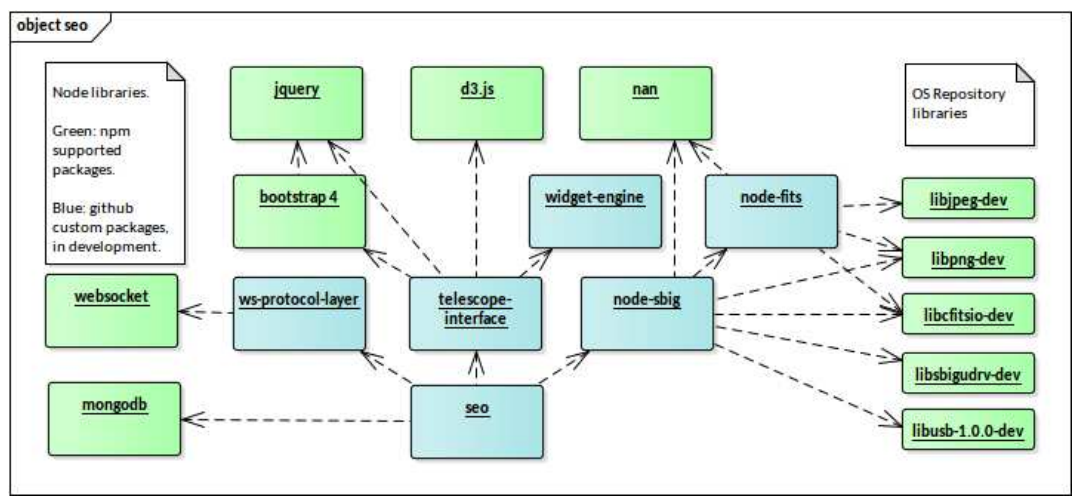

Fig. 4. Class diagram of the NODE.Js test software. In blue, the custom packages under development and available via GitHub (see text). In green, standard packages available via the NPM package manager.

by using a laptop ethernet-connected to a local modem/router/switch, which provides both internet connection, and LAN access to several devices: 1 . the dome Raspberry Pi via ssh to move the dome; 2 . the TCS pc via ssh to move the telescope; 3 . the PC in the telescope fork via web browser and websocket to control the SBIG STL 11000M camera detector and filter wheel.

In particular, we implemented the detector control software using NODE.Js, a server-side javascript runtime environment. NODE.JS allows to extend the javascript engine built-in functions and objects with new custom function and objects written in $\mathrm{C}++$ and dynamically linked to its interpreter.

In order to test the capabilities of this technology for our purposes, the SBIG STL 11000M camera NODE.JS driver and FITS file manager were developed in two separate modules by using this paradigm, while the web interface and visualization was implemented thanks to standard and widely used htm15 and javascript libraries, plus WebGL (Fig. 4). All the developed packages are available in our GitHub repository (ORSA 2017).

In the framework of OARPAF observatory and instrumentation upgrades, we want to use NODE.JS and web technologies to develop the control software of the new instrument and the related detectors. Dome and TCS will also be directly interfaced with the instrument software by using specific modules. Finally, the javascript-based, non-relational mongodb will be used as database management system to archive instrument status, logs, and FITS file position in a RAID-5 storage device Sprimont et al. (2014).

This allows to control instrument, detector, telescope, and dome, with a simple web browser, simplifying the remotization process, taking advantage from the widely support of the components by professional companies and the user community at large, and from the fact that web browser are indeed the most reliable end-user component of every OS-based device.

In order to ensure the security of web sessions and transmitted data, we plan to use a virtual private network (VPN), thanks to the implementation performed by the Liguria Digitale company.

\section{CONCLUSIONS}

In this proceedings we presented the current observatory, instrumentation, and software status at OARPAF, and upgrades are in progress in order to overcome current limitations. The dome is being substituted, the telescope maintenance is already programmed in next months, as well as the new LAN and internet connection set up. A new instrument providing photometry, long slit, and échelle spectroscopy is in a preliminary design phase. A webbased control software to control this new instrument, its detectors, the telescope, and the dome, has been recently tested on a software prototype. It can be foreseen that a full remotization will be completed by the end of this year if no further delays intervene.

Acknowledgements: We thank Antola Regional Reserve for financial support.

\section{REFERENCES}

Federici, A., Arduino, P., Riva, A., \& Zerbi, F. M. 2012, ASInC, 7, 7

ORSA. 2017, GitHub repository, https://github.com/ orsa-unige, [Online; accessed 26-November-2019]

Ricci, D., Sada, P. V., Navarro-Meza, S., et al. 2017, PASP, 129, 064401

Righi, C. 2016, Nuovo Cimento C Geophysics Space Physics C, 39, 284

Sprimont, P. G., Ricci, D., \& Nicastro, L. 2014, RMxAC, 45,75 